\title{
LETTER \\ Face Verification Based on the Age Progression Rules
}

\author{
Kai FANG ${ }^{\dagger a)}$, Member and Shuoyan LIU ${ }^{\dagger}$, Nonmember
}

SUMMARY Appearance changes conform to certain rules for a same person, while for different individuals the changes are uncontrolled. Hence, this paper studies the age progression rules to tackle face verification task. The age progression rules are discovered in the difference space of facial image pairs. For this, we first represent an image pair as a matrix whose elements are the difference of a set of visual words. Thereafter, the age progression rules are trained using Support Vector Machine (SVM) based on this matrix representation. Finally, we use these rules to accomplish the face verification tasks. The proposed approach is tested on the FGnet dataset and a collection of real-world images from identification card. The experimental results demonstrate the effectiveness of the proposed method for verification of identity.

key words: face verification, age progression rules, Bag-of-Visual-Words, difference matrix

\section{Introduction}

Face verification has been the topic of in-depth research with widespread applications [1], [2]. A large amount of works [3]-[6] pursued robustness to different image conditions (i.e. illumination change, pose variation, expression, etc. over the past several years. However, face verification across age was subjected to relatively little attention. Some previous works focused on age estimation [7], [8] and age simulation [9]. Lanitis et al. [7] used a statistical model to capture the variation of facial shapes as age progression. Ramanathan and Chellappa [8] applied a face growing models for face verification tasks for people under the age of 18. Biswas et al. [9] studied feature drifting on face images among different ages and applied it to face verification tasks. Other studies [10]-[12] used age transformation model for verification. When comparing two photos, these methods either transformed one photo to have the same age as the other, or transformed both to reduce the aging effects.

Both age estimation and age simulation are still open problems and may bring instabilities to the generative methods. Besides, these methods usually required additional information about the images being compared, such as actual age. To get around the drawback, we attempt to tackle this problem from the appearance variation aspect. The appearance changes conform to certain rules for a same person, while for different individuals the changes are uncontrolled.

To this end, this paper studies the age progression rule

Manuscript received September 5, 2014.

Manuscript revised December 23, 2014.

Manuscript publicized January 26, 2015.

${ }^{\dagger}$ The authors are with Institute of Computer Technology, China Academy of Railway Sciences, Beijing, China.

a) E-mail: 06112062@bjtu.edu.cn

DOI: 10.1587/transinf.2014EDL8178 to tackle the face verification task. The age progression rules are discovered in the difference space of facial image pairs. Based on the Bags-of-Visual-Words (BoVW) model, we first represent the image pair as a matrix whose elements are the difference of a set of visual words. Thereafter, the age progression rules are trained using Support Vector Machine (SVM) based on this matrix representation. Finally, we use these rules to accomplish the face verification tasks. The proposed approach is tested on the FGnet dataset and a collection of real-world images from identification card. The experimental results demonstrate the effectiveness of the proposed method for verification of identity.

The rest of the paper is organized as follow. Section 2 gives the proposed framework in detail. We show the performance of the proposed method on two challenging datasets in Sect. 3. Finally, Sect. 4 concludes the paper.

\section{Face Verification Based on Age Progression Rules}

The input of this system is a pair of facial images. The key idea is to discover the aging progression rules that have been used to determine the appearance changes between the two images belong to the same person (intra-personal pair) or to the different persons (extra-personal pair). An overview of the architecture is shown in Fig. 1. The first step is to construct face typical representation using the BoVW model. Given an input image pair $\left(I_{1}, I_{2}\right)$, we later represent them as a transfer matrix, which results in using of the ratios of pair-wise visual words. At the end of the process, the SVM approach is introduced to form the age progression rules, which can be used for the face verification task. Each level

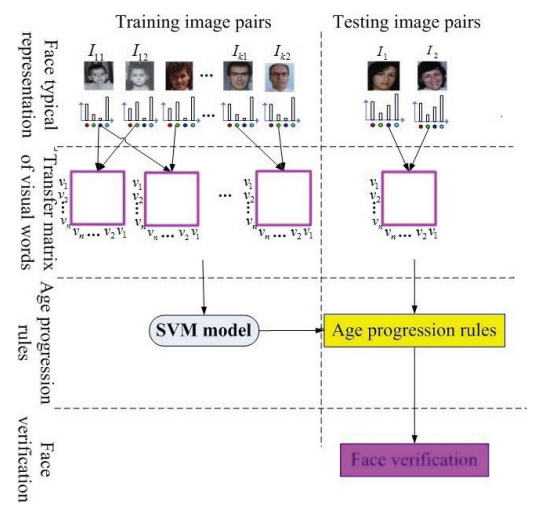

Fig. 1 Work flow of the proposed approach, where $v_{i}$ denote a discrete visual word. 


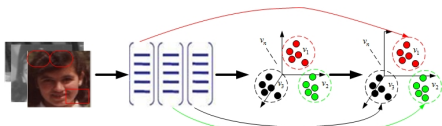

Fig. 2 The bag-of-visual-words model.

of this model will be discussed in the following in detail.

\subsection{The Face Typical Representation}

Since the imaging conditions themselves lead to challenges from difference in pose, expression and illumination, this paper extracts the Scale Invariant Feature Transform (SIFT) descriptors [13] for face image. SIFT is invariant to image scale and rotation, and is shown to provide robust matching across a substantial range of affine distortion, change in $3 \mathrm{D}$ viewpoint, addition of noise, and change in illumination. The face typical representation is designed following the bag-of-visual-words model [14]. Figure 2 illustrates the process of the bag-of-visual-words model. In special, we first use K-means to perform the clustering on all the patches, with the number of clusters provided first. After obtaining the clusters, each local SIFT descriptor in an image is further quantized into one of the clusters according to the nearest neighbor rule. Denote each cluster as a visual word, we could obtain a collections of discrete visual words $V=\left\{v_{i}\right\}_{i=1}^{n}$, where $n$ is the number of visual words. Thus a given image is represented a histogram $H=\left\{h_{i}\right\}_{i=1}^{n}$, where the bin $h_{i}$ is the value of the $i^{\text {th }}$ visual word.

\subsection{The Difference Matrix of Visual Words}

The age progression rules are discovered based on the appearance change of the two images. Hence, it needs construct the difference space of image pairs. The paper [15] utilizes the subtraction of feature space. There are two main shortages. First, some facial attributes (such as the eyes, nose etc.) are unconsidered when the visual features of face space with similar values. In addition, the verification performance is easily affected by some related issues (including illumination, pose, and facial hair).

To address the above two issues, we propose using binratio information to construct the difference space. Binratios are defined as the ratios between bin values of histograms. Such definition apparently suffers from the instability problem when bin value is close to zero. To avoid this problem, we calculate the inverse trigonometric function of each ratio. Specifically, given the two $n$-bin histograms $h$ and $g$, we define their ratio matrix $T \in \mathbf{R}^{n \times n}$ as:

$$
\begin{aligned}
& T=\left(\arctan \frac{h_{i}}{g_{i}}\right)_{i, j} \\
& =\left(\begin{array}{cccc}
\arctan \frac{h_{1}}{g_{1}} & \arctan \frac{h_{2}}{g_{1}} & \cdots & \arctan \frac{h_{n}}{g_{1}} \\
\arctan \frac{h_{1}}{g_{2}} & \arctan \frac{h_{2}}{g_{2}} & \cdots & \arctan \frac{h_{n}}{g_{2}} \\
\vdots & \vdots & \ddots & \vdots \\
\arctan \frac{h_{1}}{g_{n}} & \arctan \frac{h_{2}}{g_{n}} & \cdots & \arctan \frac{h_{n}}{g_{n}}
\end{array}\right)
\end{aligned}
$$

where $h_{i}$ denotes the $i^{\text {th }}$ elements of $h, g_{j}$ denotes the $j^{\text {th }}$ elements of $g$. In this matrix, each element $\arctan \left(h_{j} / h_{i}\right)$ measures the difference between $h_{i}, h_{j}$ in the original histogram $h$.

Thereafter we construct the difference space according to Eq.(1). The difference space comprises intra-personal image difference space and extra-personal image difference space. Let $h_{1}, g_{1}, h_{2}, g_{2}, \ldots, h_{M}, g_{M}$ be the set of $n \times 1$ vectors formed by the BoVW model in each of the $M$ pairs of faces image. The intra-personal image difference space $\left\{t_{i}\right\}_{i=1}^{M}$ (i.e., $i_{1}$ and $i_{2}$ from the same people) are obtained by the matrix of the same individual.

$$
t_{i}=h_{i} / g_{i} \quad 1 \leq i \leq M
$$

The extra-personal image difference space $\left\{t_{e}\right\}_{i=1}^{M}$ (i.e., $i_{1}$ and $i_{2}$ from different individuals) are obtained by matrix of different individuals.

$$
t_{e}=h_{i} / g_{j}, j \neq i, 1 \leq i, j \leq M
$$

According to the above equations, the bin-ratio information can reserve all facial information in the construction the difference space, even these visual words with similar values. However, the method [15] suffered from the problem. The main reason is that the paper [15] constructed the difference space by the subtraction of bin values of histogram. This setting discards the bins when their corresponding visual words with similar values. Nevertheless, each bin in the histogram corresponds to a facial attribute (such as the eyes, nose etc.). Different from [15], the binratio information is obtained by the ratios between bin values of histogram. The bin-ratio information is close $\pi / 4$ based on Eq.(1) when faced two visual words with similar bin-values. Therefore, the ratios defined this way are robust to some related issues as well as capture difference information between all pairs of bins.

\subsection{Face Verification Based on the Age Progression Rules}

We train age progression rule using Support Vector Machine (SVM) with an RBF kernel [16]. The age progression rule is separating boundary, which divides the feature space into two classes, one for intra-personal pairs and the other for extra-personal pairs. We denote the separating boundary with the following equation:

$$
\sum_{i=1}^{N_{s}} \alpha_{i} y_{i} K\left(s_{i}, x\right)+b=\Delta
$$

where $N_{s}$ is the number of support vectors and $s_{i}$ is the $i^{t h}$ support vector. $K(.,$.$) is the kernel function that provides$ SVM with nonlinear abilities. $\alpha_{i}$ is Lagrange multiplier. Each $x_{i}$ is $M$-dimensional feature vector in the image difference space. $y_{i}$ is either 1 or -1 , indicating the class to which the point $x_{i}$ belongs. In our experiments, we use the libsvm library [16] to train the age progression rule.

The face verification is modeled as a two-class classification problem. Given an input image pair $\left(I_{1}, I_{2}\right)$, matrix $T$ 
is obtained by the methods above. The face images $I_{1}$ and $I_{2}$ are the same person if matrix $T$ is separated into the positive samples (i.e. intra-personal) based on the age progression rule.

\section{Experiment Results}

We start our experiments with an in-depth analysis of our method on the FGnet dataset [17]. In addition, a study of the verification performance as a function of parameter is presented. And then the proposed approach is compared with existing methods. After that we use all of the FGnet images as training data to test the proposed method on a collection of identification card images.

The FGnet Aging Database is widely used for research of age-related facial image analysis. The database contains 1002 images from 82 subjects, over large age ranges. Consequently, there is an average of 12 images per subject in the FGnet database. This property makes the FGnet very useful for age progression study. Some examples of the FGnet dataset are shown in Fig. 3. For verification tasks, we generate 665 intra-personal pairs by collecting all image pairs from the same subjects. Extra-personal pairs are randomly selected from images from different subjects. Three-fold cross validation is used, such that in each fold images from the same subject never appear in both training and testing pairs.

Table 1 shows the confusion table which is used to illustrate the performance of our approach. In the confusion table, the $\mathrm{x}$-axis represents the results of the proposed approach. The y-axis represents the ground truth. Hence, main diagonal line gives the proportion of samples correctly predicted. Data on non-main diagonal lines indicates the proportion of the sample which is not correctly predicted. From Table 1, the average verification rate of our approach is $91.8 \%$, where the number of visual words equals to 50 . A closer look at the confusion table reveals that the higher error occurs in the extra-personal. The main reason is complex distribution in various age groups and age gaps for extrapersonal space.

We subsequently investigate how the verification performance is affected by the number of visual words. Figure 4 shows this performance variation for the four differ-

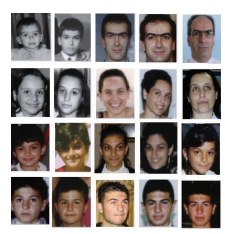

Fig. 3 The verification performance of various visual words.

Table 1 The confusion table based on the age progression rules.

\begin{tabular}{|c|c|c|}
\hline & intra-personal pair & extra-personal pair \\
\hline intra-personal pair & $92.4 \%$ & $7.6 \%$ \\
\hline extra-personal pair & $8.8 \%$ & $91.2 \%$ \\
\hline
\end{tabular}

ently sized visual words $\{10,30,50,70$ and 90$\}$. It can be seen that the performance increases progressively until visual words size is 50 , and then drops off slightly. It demonstrates that if the number of visual words is too small, it is difficult to find the age progression rules of visual words. If the number of visual words is too large, some similar face images will be match to different ones, which decrease the verification performance.

Table 2 compares verification rate of the proposed method with Ramanthan [8] on FGnet dataset. To make comparison fair, we re-implement the proposed method in the four age difference categories follows [8], i.e. 0-2 years, 3-4 years, 5-7 years, 8-9 years. From the Table 2, the proposed approach is better than the approach of Ramanthan [8] in all four age categories. Since not all samples fit to Gaussian distribution, it is not reasonable that Ramanthan [8] assumes the face difference space meets Gaussian distribution.

Train ticket real-name system is the important application for the face verification. We construct a new data set of real-world image pairs acquired from the identification card and mugshot images. To evaluate the validity of the proposed face verification algorithm in the railway ticket real-name system, we use all of the FGnet images as training data, and test the proposed approach on the real-world image pairs. Figure 5 shows some samples, where the (a) shows error verification sample, and (b) is the right one. In particular, the left image of each image pair is mugshot image, the right one is identification card image.

The average performance of this approach achieves $88 \%$ is given in Fig. 6. It is well understood that variation in pose and expression and, to a lesser extent, lighting cause significant difficulties for recognizing the identity of a person. Nevertheless, the algorithm can better complete the verification task. Further improvement is expected with

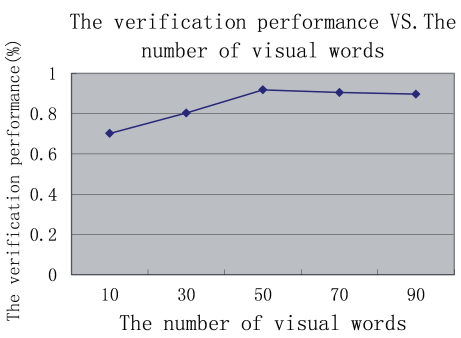

Fig. 4 The verification performance of various visual words.

Table 2 The confusion table based on the age progression rules.

\begin{tabular}{|l|c|c|c|c|}
\hline Approach & 0-2years & 3-4years & 5-7years & 8-10years \\
\hline Our approach & 0.867 & 0.943 & 0.901 & 0.923 \\
\hline Ramanthan [8] & 0.51 & 0.37 & 0.61 & 0.86 \\
\hline
\end{tabular}
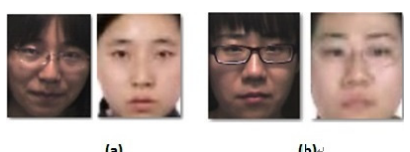

(b)

Fig. 5 The example of train ticket real-name system. 


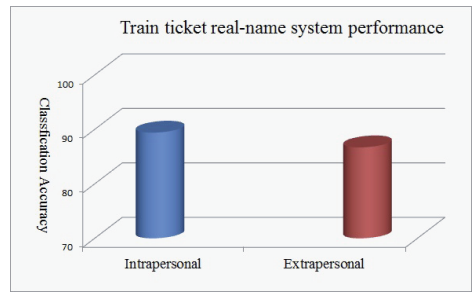

Fig. 6 Performance of train ticket real-name system.

more training data. Although the results presented here are encouraging, this task is complex, and there is a need for further improvement.

\section{Conclusion}

This paper studies age progression rule to tackle the face verification. The proposed approach has been tested on the FGnet dataset and a collection of real-world images from identification card. The experimental result has shown that the proposed algorithm can accomplish face verification effectively, even in the circumstance with low image quality, uncontrollable light and brightness. Further efforts plan to take other cognitive knowledge related with age progression into consideration to improve the verification performance.

\section{References}

[1] W. Zhao, R. Chellappa, P.J. Phillips, and A. Rosenfeld, "Face recognition: A literature survey," ACM Computer Survey, vol.35, no.4, pp.399-458, 2003.

[2] Y. Fei, Face Recognition Technology for confirmation task, Graduate School of the Chinese Academy of Sciences, 2006.

[3] Y. Fu and T.S. Huang, "Human age estimation with regression on discriminative aging manifold," IEEE Trans. Multimedia, vol.10, no.4, pp.578-584, 2008.

[4] X. Geng, Z.H. Zhou, and K. Smith-Miles, "Automatic age estimation based on facial aging patterns," IEEE Trans. Pattern Anal. Mach. Intell., vol.12, no.29, pp.2234-2240, 2007.

[5] G. Guo, Y. Fu, C.R. Dyer, and T.S. Huang, "Image-based human age estimation by manifold learning and locally adjusted robust regression," IEEE Trans. Image Process., vol.7, no.17, pp.1178-1188, 2008.

[6] G. Guo, G. Mu, Y. Fu, and T.S. Huang, "Human age estimation using bio-inspired features," Proc. IEEE Conf. Computer Vision and Pattern Recognition (CVPR), pp.112-119, 2009.

[7] A. Lanitis, C.J. Taylor, and T.F. Cootes, "Toward automatic simulation of aging effects on face images," IEEE Trans. Pattern Anal. Mach. Intell., vol.24, no.4, pp.442-455, 2002.

[8] N. Ramanathan, "Face verification across age progression," Proc. IEEE Conf. Computer Vision and Pattern Recognition, pp.462-469, 2005.

[9] S. Biswas, G.N.R. Aggarwal, and R. Chellappa, "A non-generative approach for face recognition across aging," Proc. IEEE Conf. Biometrics: Theory, Applications and Systems, pp.1-6, 2008.

[10] X. Geng, Z.Z. Hua, and K.M. Smith, "Automatic age estimation based on facial aging patterns," IEEE Trans. Pattern Anal. Mach. Intell., vol.12, no.29, pp.2234-2240, 2007.

[11] J. Suo, X. Chen, S. Shan, and W. Gao, "Learning long term face aging patterns from partially dense aging databases," Proc. Int. Conf. Computer Vision (ICCV'09), pp.622-629, 2009.

[12] U. Park, Y. Tong, and A.K. Jain, "Age-invariant face recognition," IEEE Trans. Pattern Anal. Mach. Intell., vol.32, no.5, pp.947-954, 2010.

[13] D. Lowe, "Distinctive image features from scale-invariant key points," Int. J. Comput. Vis., vol.60, no.2, pp.91-110, 2004.

[14] E. Nowak, F. Jurie, and B. Triggs, "Sampling strategies for bag-offeatures image classification," Proc. European Conference on Computer Vision (ECCV'06), pp.490-503, 2006.

[15] H. Ling, S. Soatto, and N. Ramanathan, "Face verification across age progression using discriminative methods," IEEE Trans. Information Forensics and Security, vol.1, no.5, pp.82-91, 2010.

[16] C. Chang and C. Lin, "LIBSVM: A library for support vector machines," available from http://www.csie.ntu.edu.tw/ cjlin/libsvm.

[17] Face and Gesture Recognition Working Group 2000 [Online]. Available: http://www-prima.inrialpes.fr/FGnet/ 